\title{
adegraphics: An S4 Lattice-Based Package for the Representation of Multivariate Data
}

\author{
by Aurélie Siberchicot, Alice Julien-Laferrière, Anne-Béatrice Dufour, Jean Thioulouse and Stéphane \\ Dray
}

\begin{abstract}
The ade4 package provides tools for multivariate analyses. Whereas new statistical methods have been added regularly in the package since its first release in 2002, the graphical functions, that are used to display the main outputs of an analysis, have not benefited from such enhancements. In this context, the adegraphics package, available on CRAN since 2015, is a complete reimplementation of the ade4 graphical functionalities but with large improvements. The package uses the S4 object system (each graph is an object) and is based on the graphical framework provided by lattice and grid. We give a brief description of the package and illustrate some important functionalities to build elegant graphs.
\end{abstract}

\section{Introduction}

In many fields, data consists in tables containing measurements of several variables for a number of samples. In this context, multivariate analyses provide tools to summarize the main structures of multivariate data. After a dimension reduction step, these methods provide a graphical display of the primary relationships between variables and similarities between samples. Multivariate methods are routinely used in various fields including marketing, psychometry or ecology and have been implemented in several R packages (e.g. vegan (Oksanen et al., 2017), MASS (Venables and Ripley, 2002), FactoMineR (Lê et al., 2008), see the Multivariate Task View of CRAN). The ade4 package (Dray and Dufour, 2007) is an alternative, distributed on CRAN since 2002, that implements more than 30 different methods, mainly oriented to the analysis of ecological data, and around 40 graphical functions to display the results of analyses.

During the last 15 years, a major effort was made in the implementation of new statistical methods in ade4 but the graphical functionalities have not benefited from such improvements. At this time, graphs from ade4 were black and white and lacked flexibility. It became increasingly difficult to customize the graphical outputs as the results of analyses are increasingly complex. In parallel, $\mathrm{R}$ has evolved and now proposes new paradigms of development (the S4 object system) and new graphical environments (lattice (Sarkar, 2008), grid (Murrell, 2005)). In this context, we started the development of a new add-on package: adegraphics. It is available on CRAN since 2015 and is designed to provide enhanced graphical functionalities for representing outputs of multivariate analysis, especially from ade4.

For users, adegraphics provides a flexible environment to produce, edit and easily manipulate graphs. To ensure continuity with the graphical functions of ade4, functions and parameters names have been largely preserved in adegraphics, so that regular users can easily migrate to the new package. Moreover, the graphical functions of ade4 are not removed to preserve the functioning of other $\mathrm{R}$ packages and scripts that depend on ade4. The use of the new adegraphics functions is preferred and encouraged for future developments. Keeping the old version of the functions in ade4 has some practical effects. In a classical case, when multivariate analyses are performed with ade4, it is imperative to load ade4 before adegraphics. This solves the issues of conflicting names between functions by ensuring that the versions from adegraphics will be used.

\footnotetext{
$>$ library(ade4)

$>$ library (adegraphics)
}

The adegraphics package can be installed from CRAN. A development release is available on GitHub (https://github.com/sdray/adegraphics) and a detailled description of the package is given in the vignette available at https://cran.r-project.org/web/packages/adegraphics/vignettes/ adegraphics. html or in any $\mathrm{R}$ session by typing vignette("adegraphics"). A reproducible version of the code in this paper is available online at http://lbbe-shiny.univ-lyon1.fr/Reproducible Research/TRJ. Siberchicot.2017/.

This paper is divided in three parts: simple graphs, multiple graphs, and multivariate analysis graphs. The simple graphs part includes a presentation of basic elements and graphical parameters, with examples of graph manipulation and spatial representations. The multiple graphs part shows how to do automatic collections of graphs, and how to create and customize multiple graphs. The 
multivariate analysis part shows an example of how the plot of a coinertia analysis can be customized and improved with adegraphics.

\section{A simple example}

We illustrate some principles and functionalities of adegraphics by considering the analysis of the mafragh data set available in ade4. It contains a phyto-ecological survey collected in an Algerian plain, called La Mafragh (de Bélair and Bencheikh-Lehocine, 1987; Pavoine et al., 2011).

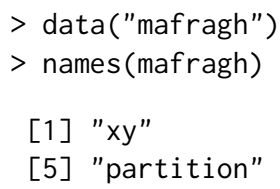

The aim of the original study (de Bélair and Bencheikh-Lehocine, 1987) was to propose an integrated management project of the Mafragh marshy coastal plain. mafragh $\$ f l o$ is a data frame giving the abundance of 56 plant species (columns) in 97 sites (rows) distributed in the plain. mafragh\$env is a data frame with 97 rows and 11 columns describing the physico-chemical and topographic characteristics, and the granulometry of soil samples taken at the same sampling sites. mafragh\$partition is a factor defined by de Bélair and Bencheikh-Lehocine (1987), classifying the 97 sites in 7 ecologically coherent regions according to observed vegetation units (ecoregions).

\section{Basic elements and simple graphs}

\section{Classes, objects and calling functions}

The adegraphics package uses object-oriented programming (OOP) in R: graphs are S4 objects that can be displayed or stored for later modification.

In adegraphics, there are two main parent classes of objects, one to store simple graphs (i.e. with only one represented information) called "ADEg" and the other, called "ADEgS", to store multiple graphs. The "ADEg" class has 5 sub-classes dedicated to different representation types: the "ADEg.T" class can be used to represent raw data such as distance matrices or contingency tables, the "ADEg.S2" class is associated with bidimensional data and can show factorial maps, the "ADEg.S1" class is used to represent a numeric score such as only one factorial axis in a unidimensional graph, the "ADEg.C1" class allows to represent unidimensional data associated with a supplementary information into two dimensions and the "ADEg.Tr" class is a peculiar class to represent data in a ternary plot. Each of these sub-classes itself has several child classes. The list of classes evolves and can be enriched to satisfy future developments. The adegraphics package provides user-friendly functions to create each type of graphical object. Some examples of these functions are listed in the Table 1 for each of the 5 sub-classes.

\begin{tabular}{|c|c|c|}
\hline "ADEg" sub-classes & Example of sub-classes & Example of associated user functions \\
\hline "ADEg.T" & “T.image", “T.value", ... & table. image, table.value,... \\
\hline "ADEg.S2" & $\begin{array}{l}\text { "S2.class", "S2.corcircle", } \\
\text { "S2.density", "S2.value", ... }\end{array}$ & $\begin{array}{l}\text { s.class, s. corcircle, } \\
\text { s.density, s.value,... }\end{array}$ \\
\hline "ADEg.S1" & $\begin{array}{l}\text { "S1.boxplot", “S1.distri", } \\
\text { "S1.match", .. }\end{array}$ & $\begin{array}{l}\text { s1d.boxplot, s1d.distri, } \\
\text { s1d.match,... }\end{array}$ \\
\hline "ADEg.C1" & $\begin{array}{l}\text { "C1.curve", "C1.gauss", } \\
\text { "C1.hist", .. }\end{array}$ & $\begin{array}{l}\text { s1d. curve, s1d.gauss, } \\
\text { s1d.hist,... }\end{array}$ \\
\hline "ADEg.Tr" & $\begin{array}{l}\text { "Tr.class", "Tr.label", } \\
\text { "Tr.match", "Tr.traject" }\end{array}$ & $\begin{array}{l}\text { triangle.class, triangle. label, } \\
\text { triangle.match, triangle.traject }\end{array}$ \\
\hline
\end{tabular}

Table 1: Examples of classes and associated graphical functions in adegraphics

In our example, a normed principal component analysis (PCA) is applied (using the dudi . pca function of ade4) on the mafragh $\$$ env data table. This table contains the measurements of 11 environmental variables for 97 sites. The first four axes are kept and the results are stored in object pca1. 
$>$ pca1 <- dudi.pca (mafragh\$env, scale $=$ TRUE, center $=$ TRUE, scannf $=$ FALSE, $\mathrm{nf}=4)$

The sites can be displayed on the factorial map formed by the first two axes. A graphical object is created (by the call to the s.label function) but not printed because of the argument plot = FALSE. Correlations between environmental variables and the first two PCA axes are represented on a correlation circle (with the user function s. corcircle, Figure 1). Both graphs (g_sl1 and g_sc1) are stored in $\mathrm{S} 4$ objects of different sub-classes.

$>$ g_sl1 <- s.label $($ pca1\$li, plot $=$ FALSE $)$
$>$ class $($ g_sl1 $)$

[1] "S2.label"

attr (, "package")

[1] "adegraphics"

$>$ g_sc1 <- s.corcircle $($ pca1\$co)

$>\operatorname{class}($ g_sc 1$)$

[1] "S2.corcircle"

attr (, "package")

[1] "adegraphics"

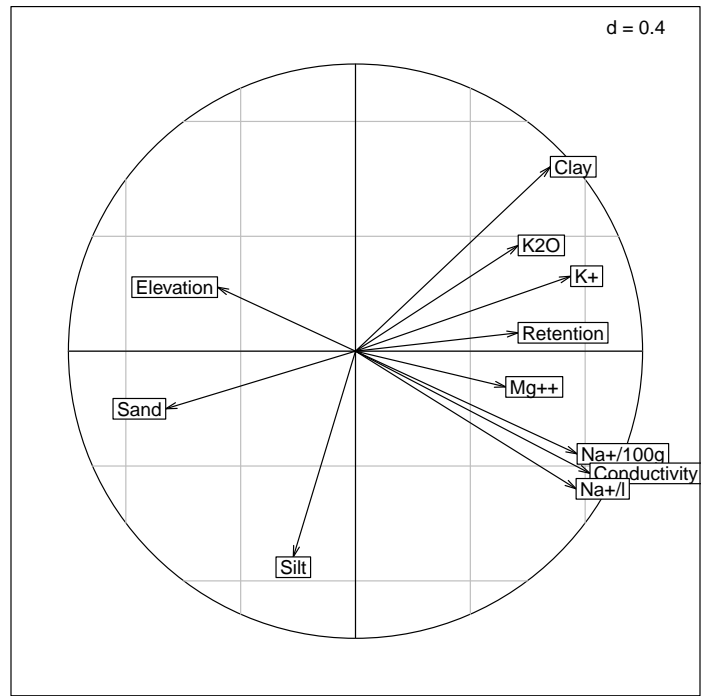

Figure 1: Default representation of the correlation circle of the 11 environmental variables on the first two PCA axes (g_sc1). The direction and length of arrows show correlations between variables and principal components. This graph helps to describe the Mafragh environment. The first axis is a salinity/elevation gradient with high salinity and low altitude on the right, opposed to high elevation and low salinity on the left. The second axis refines this gradient by opposing clay and potassium ions (up) to sodium ions and silts (down).

Each simple graphical object created with adegraphics (i.e. object belonging to class "ADEg") is defined by 8 attributes. These attributes are reachable by the @ operator and their names are given by the slotNames function.

$>\operatorname{slotNames}($ g_sc1)

[1] "data" "trellis.par" "adeg.par" "lattice.call" "g.args"

[6] "stats" "s.misc" "Call"

The data slot contains information about the data used in the plot and the Call slot contains the matched call.

$>$ g_sc1@Call

s. $\operatorname{corcircle}(d f x y=p c a 1 \$ c o, x a x=1, y a x=2$, labels $=$ row. names $(a s$. data. frame $(p c a 1 \$ c o))$, fullcircle $=$ TRUE, facets $=$ NULL, plot $=$ TRUE, storeData $=$ TRUE, add $=$ FALSE, pos $=-1$ ) 
Slots s.misc and stats store lists of internal parameters and internal preliminary computations. The lattice.call slot contains all the information required to create a "trellis" object (see the lattice package) associated to the adegraphics instruction. Note that an adegraphics object can be converted to a lattice one using the gettrellis function. The trellis. par, adeg. par and g. args slots manage several graphical parameters and are detailed in the next section.

\section{Graphical parameters}

A great flexibility is provided in adegraphics by the management of many graphical parameters. Parameter names are more intuitive and their number is greater than in ade4. There are 3 kinds of parameters in adegraphics user functions.

First, parameters implemented in adegraphics are itemized by the adegpar function (that works like the basic par function). When the graphical object is created, these parameters are stored in the adeg.par attribute. For example, the adeg.par attribute of the g_sc1 object is yielded by g_sc1@adeg.par or by getparameters (g_sc1,2). These parameters can be applied locally on a graph (as arguments to a user function) or globally in the working environment (using the adegpar function) and so applied on all graphs created thereafter.

Second, lattice parameters are managed in adegraphics and stored in the trellis.par attribute of the created object. For example, the trellis.par attribute of the g_sc1 object is returned by g_sc1@trellis.par or by getparameters $\left(g_{-}\right.$sc 1,1$)$. These parameters are itemized by the lattice function trellis.par. get and can also be applied locally or globally (see the trellis.par. set function of lattice).

Last, some general parameters are also managed like xlim and ylim, xlab and ylab, main, scales. These parameters are stored in the $g$. args attribute of the created object.

The adegraphics vignette explains more precisely how to manage these various graphical parameters and which one can be applied according to the class of the created object. The vignette appendix lists the correspondences between the graphical parameters used in ade4 and their translation in adegraphics.

In the example below, the representation of environmental variables onto the first factorial map of pca1 in the correlation circle (Figure 1) is customized: boxes around labels are removed and a subtitle is added. The background color of this new graph (called g_sc2) can be reached as the element col of the pbackground list of the adeg.par attribute. Here, the background color of g_sc2 is "white". Note that g_sc2 is not printed right now (plot = FALSE).

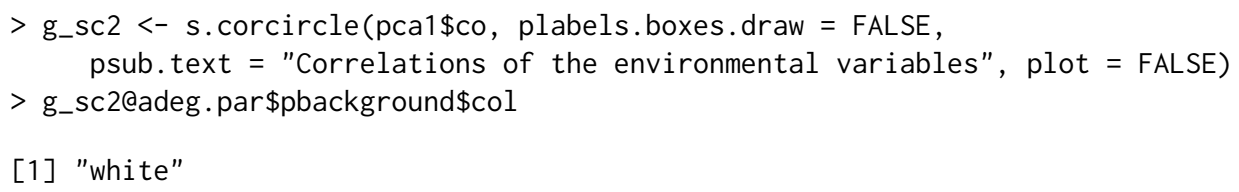

\section{Manipulating a simple graph}

There are several methods in adegraphics to manipulate the graphical objects created and stored. For example, the update method allows to modify a posteriori some graphical parameters of a graph previously created with adegraphics, without recreating it. Besides, users can zoom in or out a stored graph thanks to the zoom method. Note that this function is only allowed for some sub-classes of adegraphics. Simply, the plot, print and show methods can be used to display a stored adegraphics object. Other functions and methods are described in the vignette of the adegraphics package.

In the example below, the grid and background color of the previously created g_sc 2 object are changed using the update method (Figure 2). As above, the background color parameter is reachable through the adeg. par slot of the g_sc2 object; now it is "grey90".

$>$ update(g_sc2, pgrid.col = "white", pbackground.col = "grey90")

$>$ g_sc2@adeg.par\$pbackground\$col

[1] "grey90"

\section{Representation of spatial information}

In ade4, classes were implemented to manage spatial information: the "area" class for geographic maps and the "neig" class to store spatial neighborhood objects. The adegraphics package abandoned 


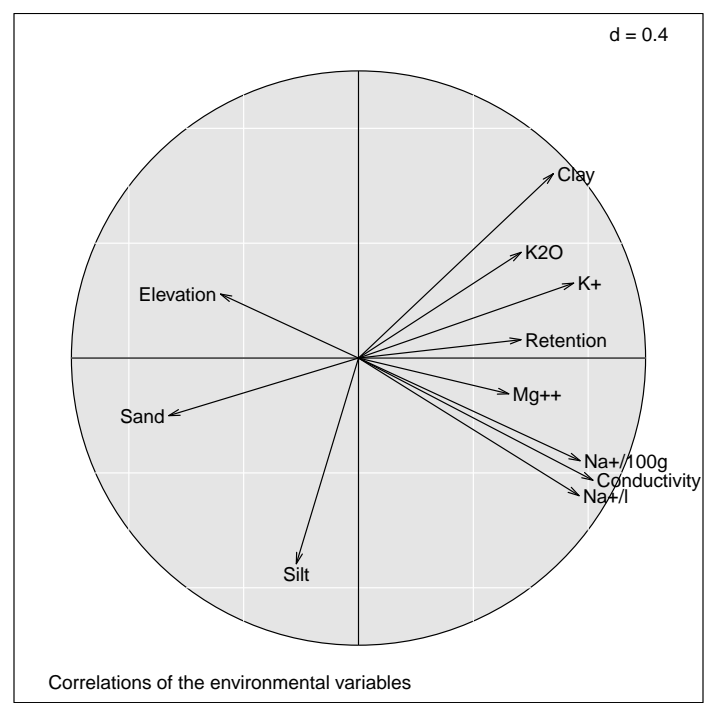

Figure 2: Customization of the g_sc2 object using the update method. Labels are more readable and the graph is highlighted by changing the colors and adding a title.

these outdated implementations and adopted more efficient classes. Maps are now considered as objects inherited from classes of the sp package (Pebesma and Bivand, 2005; Bivand et al., 2013b). "nb" and "listw" objects, from the spdep package (Bivand and Piras, 2015; Bivand et al., 2013a), are used to manage spatial neighborhood graphs. Note that only "ADEg.S2" objects (bidimensional plot of xy coordinates, implemented in s.* functions) of adegraphics support the representation of spatial objects by the use of arguments Sp and nb. See also the useful s. Spatial function to easily draw thematic maps. adegraphics provides specific parameters to manage the customization of spatial objects: the pSp parameters affect maps and pnb affects neighborhood.

In our example, a thematic map is simply obtained by displaying the score of sites on the first PCA axis (pca1\$1i[,1]) on a spatial map used as background. The s. value function is hereafter used to create the g_sv1 object (Figure 3, left) and takes as argument the mafragh\$Spatial. contour object (an object of a class from the sp package) in the Sp parameter and a color palette in the col parameter. As many colors as classes of value are stored in a valuecolors vector created with the brewer. pal function of the RColorBrewer package (Neuwirth, 2014).

$>$ class (mafragh\$Spatial. contour)

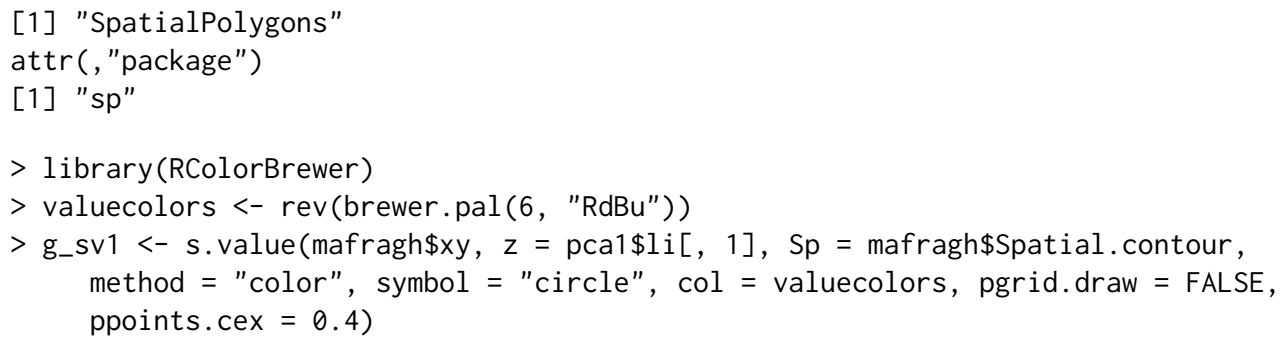

A similar graph (called g_sp1) can easily be built (Figure 3, right) using the s. Spatial function and a "SpatialPolygonsDataFrame" object (from the sp package). The spobj object couples a "SpatialPolygons" object with the scores of sites on the first PCA axis.

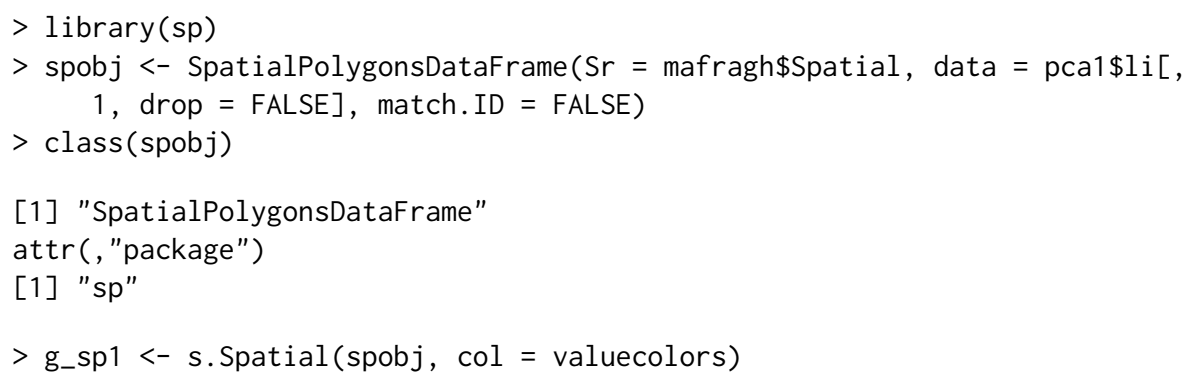




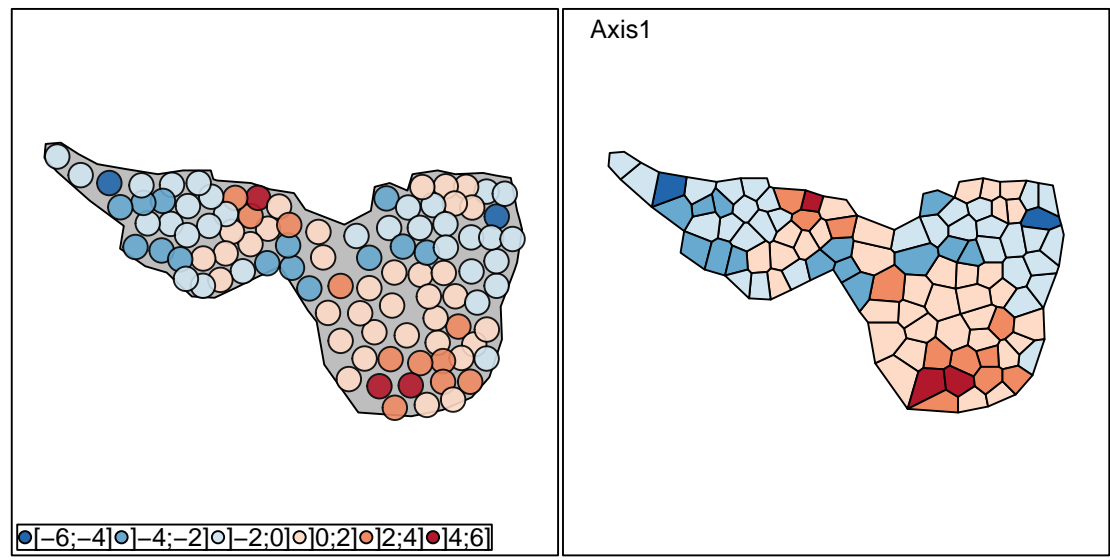

Figure 3: Thematic map: the scores on the first PCA axis are mapped in the geographical space. The g_sv1 object (left) is created by a call to the s. value function and the g_sp1 object (right) by the s. Spatial function. The color of sites is given by the value of scores on the first axis. The PCA first axis described by the correlation circle in Figure 2 helps interpret this figure: regions with high elevation (blue color, negative coordinates) are located at the extreme west and east, while the lowest points are located in the center of the map. Salinity (particularly potassium ions) is high in the southern region (red color, positive coordinates) because local conditions (clay) prevent the drainage of sea waters that accummulate there. Salinity (sodium ions) is also high in a particular region of the north because it is just next to the sea shore and receives a lot of sea water that stays trapped here.

\section{Dealing with multiple graphs}

The "ADEgS" class stores a collection of "ADEg", "ADEgS" and/or "trellis" (from the lattice package) objects, as a list of graphical objects. It is defined by 4 attributes, given by the slotNames function applied on a "ADEgS" object:

- ADEglist contains the list of graphs (i.e. objects) in the collection;

- positions is a matrix that contains the positions of subgraphs in the display;

- add is a matrix that contains the information about subgraphs superposition;

- Call contains the matching call.

"ADEgS" objects can be simply manipulated with methods associated to lists (i.e. \$, [], [[]]). The names function outputs the labels of subgraphs contained in an "ADEgS" object and the length function returns the number of subgraphs stored in the object.

We implemented different ways to generate collections of graphs. "ADEgS" objects can be created by a call to a simple function or built step-by-step using longer code. They can be customized in the same way as "ADEg" objects.

\section{Automatic collections}

Several user-friendly functionalities are implemented in adegraphics to facilitate the creation of collections of graphs, repeating (without using a for-loop command) a simple graph for different groups of individuals, variables or axes. The building of these collections is very simple for the user (definition of an argument in the call of a function) and leads to the creation of an "ADEgS" object.

For instance, as in the ggplot2 package (Wickham, 2016), a facets argument is available to split a dataset into groups of individuals and to produce one graph per group. The g_sv2 object (Figure 4) displays the scores of sites of the first PCA axis (as in the g_sv1 object, left of Figure 3) for each of the 7 ecoregions, called $\mathrm{C} 1, \ldots, \mathrm{C} 7$, defined in magragh\$partition.

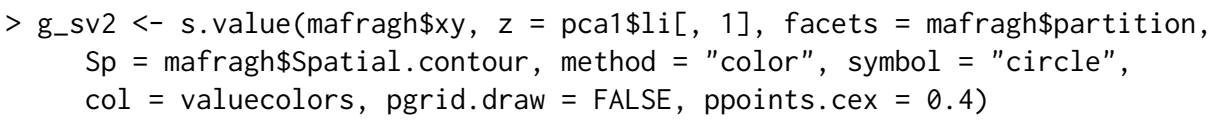

The g_sv2 object is an "ADEgS" containing 7 subgraphs belonging to the "S2.value" class. The subgraphs names are given by the names function and are equal to the level names of mafragh\$partition. Combined with the $\$$ symbol, these names can be used to select only one subgraph to the "ADEgS" object. For example, g_sv $2 \$ C 1$ is the extraction of the $\mathrm{C} 1$ subgraph of $g_{-} s v 2$. 


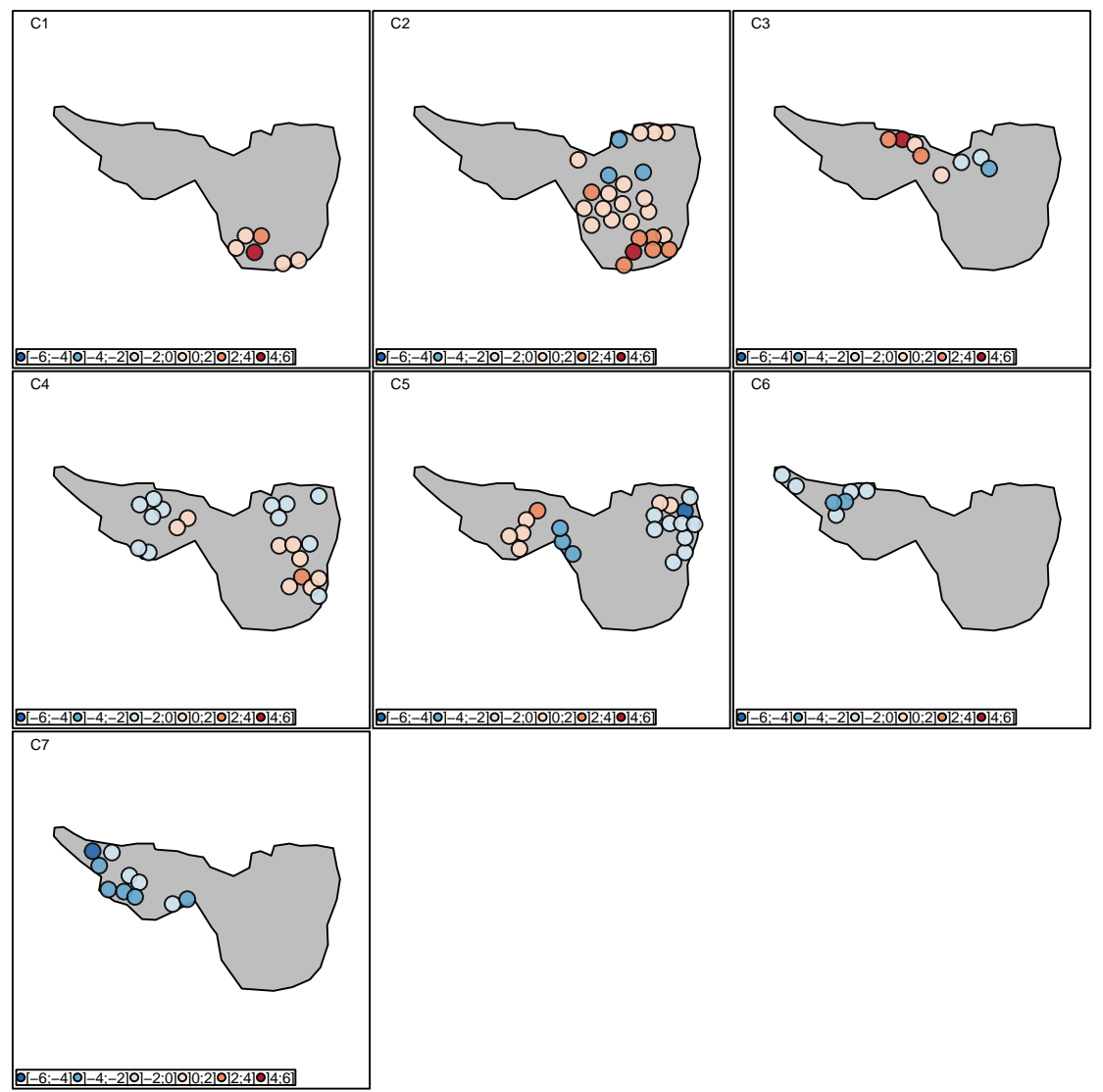

Figure 4: Automatic creation of an "ADEgS" object using the facets argument. The g_sv2 object is created by only a call to the s. value function. Seven thematic maps (one for each ecoregion) are displayed to facilitate the read of PCA results. These 7 ecoregions were defined by de Bélair and BencheikhLehocine (1987) from a correspondence analysis of the plant species data table (mafragh\$flo) and phytosociological interpretations. Each ecoregion takes into account the distribution of plant species of various floristic groups, but also pedological characteristics of sampling sites, and geographical areas. For example, ecoregion $\mathrm{C} 1$ is defined by 6 pedological sampling sites (numbered 43, 44, 45, 46, 49 and 51 in g_sv2\$C1@data $\$ \mathrm{dfxy}$ ) and the Bolboschoenus maritimus and Schoenoplectus litoralis plant communities (high abundances in mafragh $\$$ flo $[c(43,44,45,46,49,51)$,$] ).$

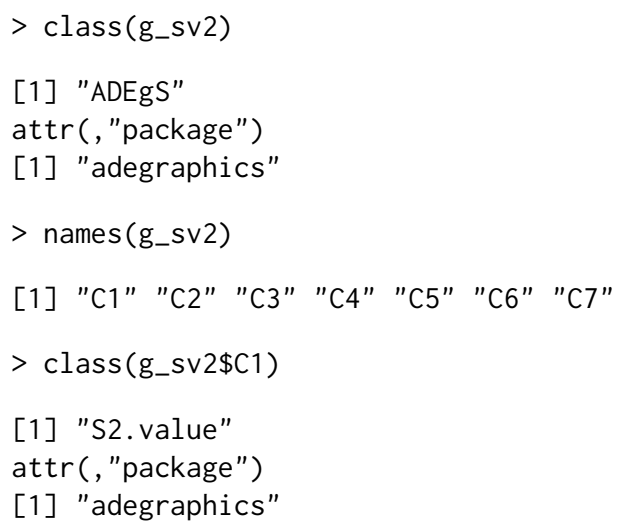

On the other hand, multiple graphs can also be produced by splitting graphs by columns. This is achieved when a data frame with several variables is given as an argument to a function that usually requires a vector. For instance, the abundance of four focused species is easily displayed along the mafragh sites. The g_sv3 object (Figure 5) is created with the s.value function, using the mafragh $\$ f l o[$, selectedspecies] data frame as $z$ parameter. A line surrounding the entire mafragh region (contained in the mafragh\$Spatial. contour object) is added thanks to the Sp argument.

$>$ selectedspecies $<-c(9,12,31,34)$

$>$ floselectedspecies <- mafragh $\$$ flo[, selectedspecies] 
$>$ colnames(floselectedspecies) <- mafragh\$spenames\$code[selectedspecies]

$>$ colnames(floselectedspecies)

[1] "Juma" "Scli" "Boof" "Plco"

$>$ g_sv3 <- s.value (mafragh $\$ x y, z=$ floselectedspecies, symbol = "circle", centerpar $=\operatorname{list}($ cex $=0.1)$, ppoints.cex $=0.7$, pgrid.draw $=$ FALSE, psub.cex $=2$, porigin.draw $=$ FALSE, plegend.drawK $=$ FALSE, $\mathrm{Sp}=$ mafragh\$Spatial. contour $)$

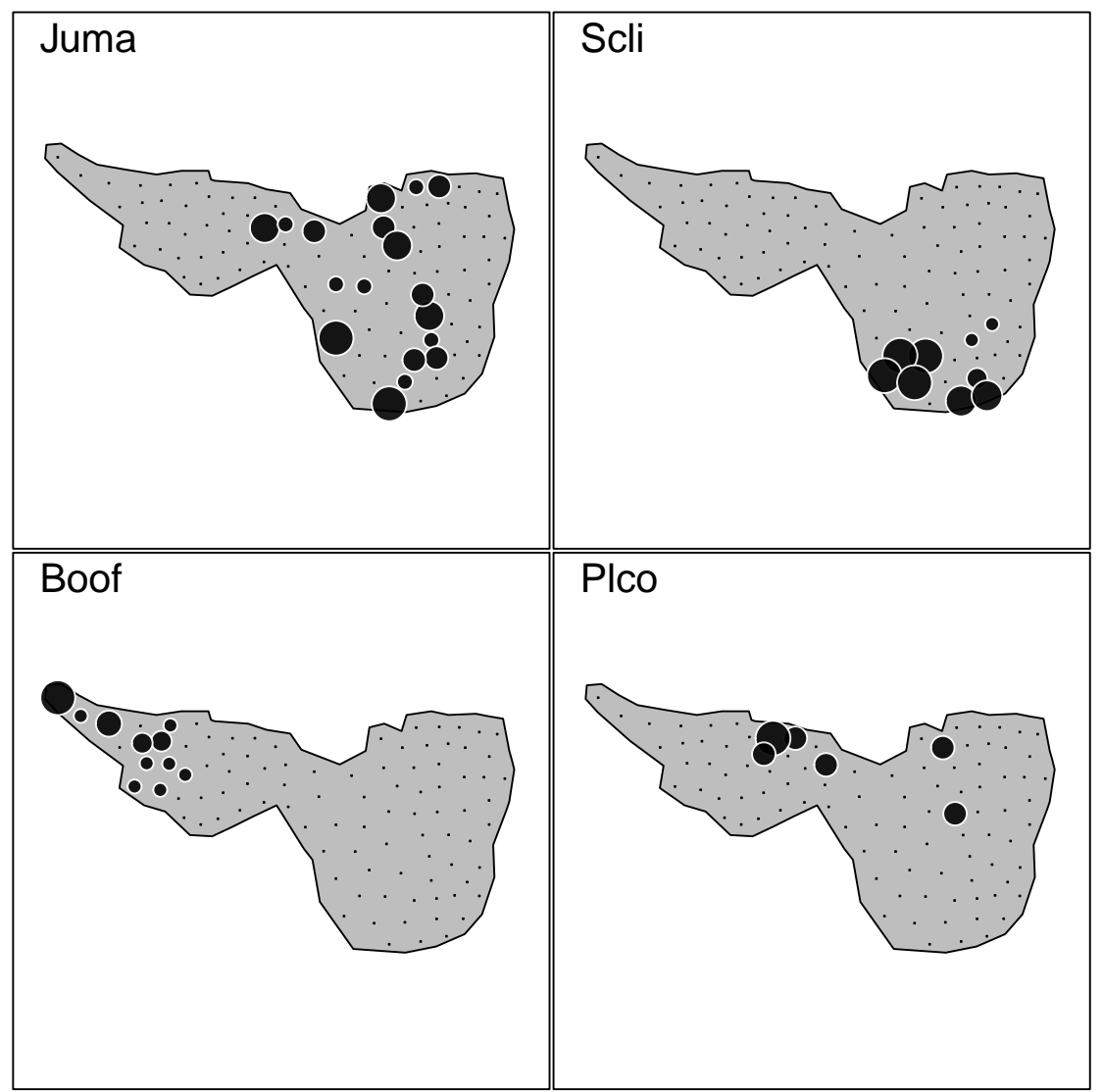

Figure 5: Automatic creation of an "ADEgS" object. The g_sv3 object is created by only a call to the $s$. value function using a multivariate argument $z$. Four maps of abundance are produced, one for each of four species of interest: Juncus maritimus (Juma), Schoenoplectus litoralis (Scli), Borago officinalis (Boof) and Plantago coronopus (Plco).

\section{Step-by-step creation}

"ADEgS" objects can also be created by the manipulation of several simple graphs. Several functions in adegraphics allow superposition, combination or insertion of two objects: superpose, +, cbindADEg, rbindADEg and insert.

Following our example, a Correspondence Analysis (CA, called coa1) is performed on the abundance of the 56 plant species of mafragh (contained in the mafragh $\$$ flo data table) with the dudi.coa function of the ade4 package, displayed but not shown with the s.label function and stored in a g_sl2 object. Note here the plabel.optim parameter set to TRUE which automatically optimize the label positions and remove label boxes.

$>$ coa1 <- dudi.coa(mafragh\$flo, scannf = FALSE, $\mathrm{nf}=2$ )

$>$ g_sl2 <- s.label (coa1\$co, labels = mafragh\$spenames $\$$ code, plabel.optim = TRUE, plot $=$ FALSE)

To help interpret the results of this CA, three among four maps of species abundance created in the g_sv3 object can be inserted, one at a time, close to the matching species point on the g_sl2 object. The insert function takes as parameters a first graph to insert, a second one in which to insert and a 
posi argument to define the insertion position. Each of the three inserted subgraph is extrated from the g_sv3 object by the $[[]]$ method. The final output called g_in1 is an object of "ADEgS" class (Figure 6).
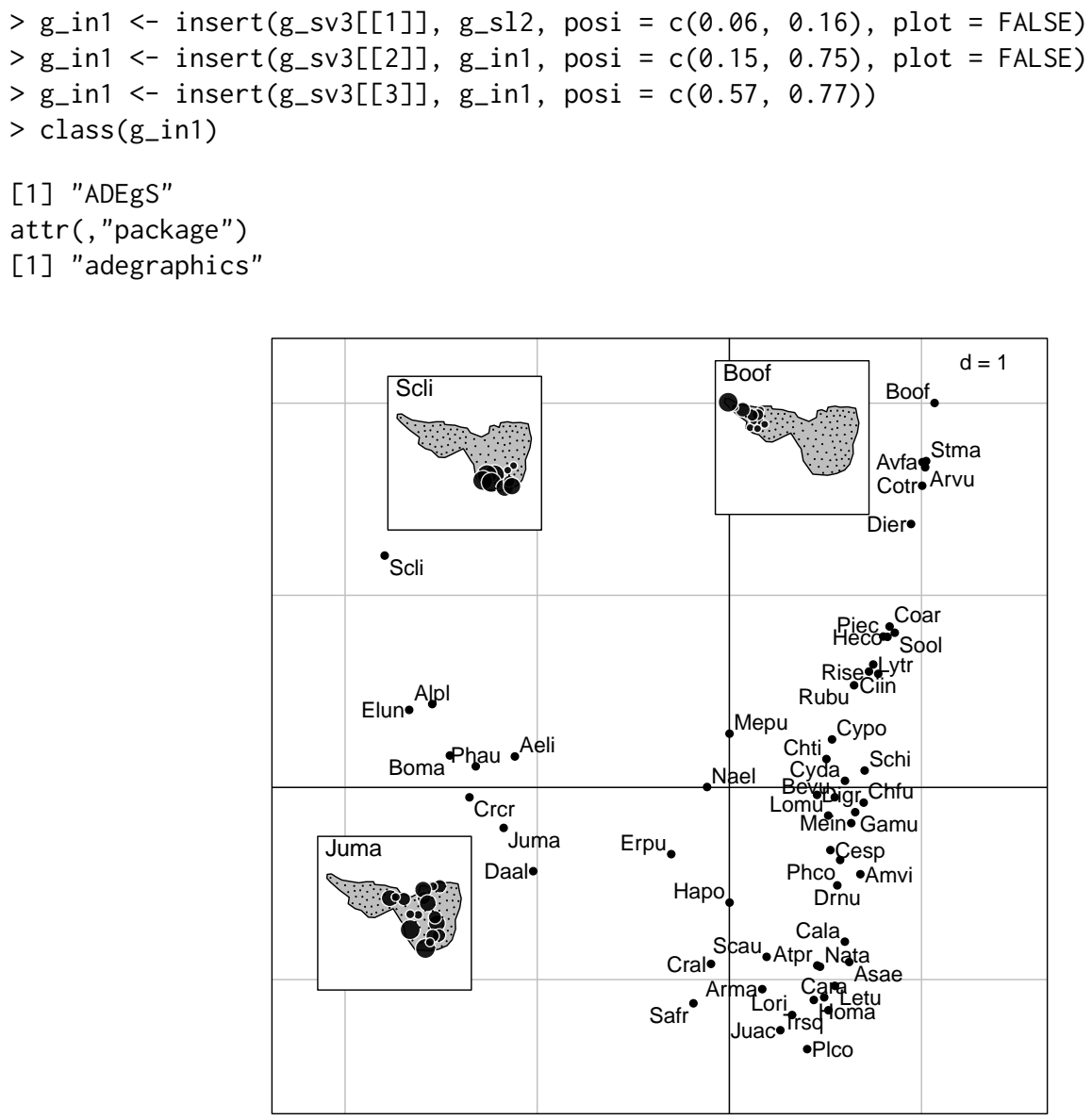

Figure 6: Creation of an "ADEgS" by the manipulation of four "ADEg" objects. This display can be produced by the insert or ADEgS function. Three geographical maps are superimposed on the first factor map of the CA of the plant species table. The spatial distribution of Juncus maritimus (Juma), Schoenoplectus litoralis (Scli) and Borago officinalis (Boof) are very different, and the CA scores clearly take these differences into account, even though spatial coordinates of sampling sites are not used in the analysis. They correspond to ecoregions $\mathrm{C} 1, \mathrm{C} 2$ and $\mathrm{C} 6$, respectively.

This object has four elements (i.e. graphs). As for a standard list, the function names can be used to modify labels of each g_in1 subgraph:

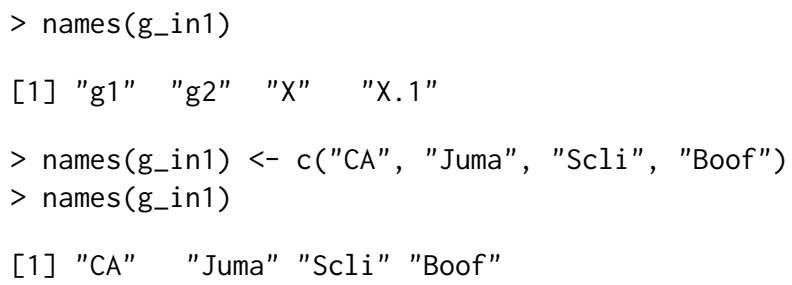

The insert function is a shortcut that calls a very flexible function designed to generate "ADEgS" objects. The general ADEgS function allows to arrange multiple graphs. It takes as arguments (i) a list of several "ADEg", "ADEgS" and/or "trellis" objects and (ii) information about their layout.

Below, the g_in2 object, created by the ADEgS function, is strictly identical to g_in1. The positions of each of the four subgraphs is yielded by the positions attribute of the g_in1 object.

\section{$>$ g_in1@positions}

$[, 1][, 2][, 3][, 4]$

0.000 .001 .001 .00 
positions $0.06 \quad 0.16 \quad 0.26 \quad 0.36$

positions 0.150 .750 .350 .95

positions $0.57 \quad 0.77 \quad 0.77 \quad 0.97$

$>$ g_in2 <- ADEgS(list $\left(C A=g_{-} s l 2\right.$, Juma $=g_{-} s v 3[[1]]$, Scli $=$ g_sv3[[2]],

Boof $\left.=g_{-} s v 3[[3]]\right)$, positions $=\operatorname{rbind}(c(0,0,1,1), c(0.06$,

$0.16,0.26,0.36), c(0.15,0.75,0.35,0.95), c(0.57,0.77$

$0.77,0.97)$ ), plot $=$ FALSE)

\section{Customizing an "ADEgS"}

Like simple graphs ("ADEg"), the multiple graphs generated with adegraphics can be customized during or after the creation of the object, thanks to the update function. It is possible to apply changes to all or only one subgraph contained in the "ADEgS". To modify a graphical parameter for all subgraphs of the "ADEgS", the syntax is the same as for a simple "ADEg". To modify a graphical parameter for only a given subgraph, the parameter name must be preceded by the name of the subgraph, separated by a dot. This supposes that the subgraph names (available by a call to the names function) is known.

For example, the background color of the g_in1 object can be updated for all subgraphs:

$>$ update (g_in1, pbackground.col = "grey90")

or only for the one called CA:

$>$ update(g_in1, CA.pbackground.col = "grey90")

The "ADEgS" created hereafter by the ADEgS function is a good example how to manipulate "ADEg" objects and deal with adegraphics graphical parameters. The g_adegs 1 object is a personal graph (Figure 7) to explore pca1 results. It arranges four "ADEg" objects in a personal layout.

First, default global graphical parameters are stored in a oldadegpar object in order to be restored at the end by the adegpar function.

$>$ oldadegpar <- adegpar()

Two graphs called g_gau1d and g_lab1d are created to represent the scores of ecoregions and environmental variables on the first pca1 axis. Parameters about unidimensional displays, affecting this two graphs, are globally modified by the call of the adegpar function.

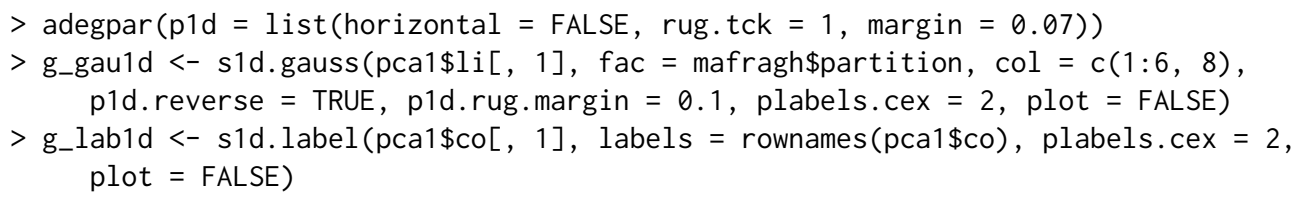

Then the thematic map of the scores of sites on the first pca1 axis (g_sv1, Figure 3 at left) is kept

Finally the graph of the pca1 eigenvalues is created (called g_eig) with the relevant plotEig function of adegraphics. Here, only the first axis is kept to interpret the results (see the black bar).

$>$ g_eig <- plotEig(pca1\$eig, xax = 1, yax = 1, nf = 1, pbackground. box = TRUE, plot $=$ FALSE)

The g_adegs 1 can now be created, arranging the four graphs previously stored.

$>$ g_adegs1 <- ADEgS(list(g_gau1d, g_lab1d, g_sv1, g_eig),

layout $=$ matrix $(\mathrm{c}(1,2,3,1,2,4)$, nrow $=2$, byrow $=$ TRUE $)$, plot $=$ FALSE $)$

After the "ADEgS" creation, some graphical parameters can be a posteriori updated: the grid is removed on all graphs, a title is added to the third (called g3 in the g_adegs 1 object) and fourth (called g4 in the g_adegs1 object) graphs and these titles are enlarged.

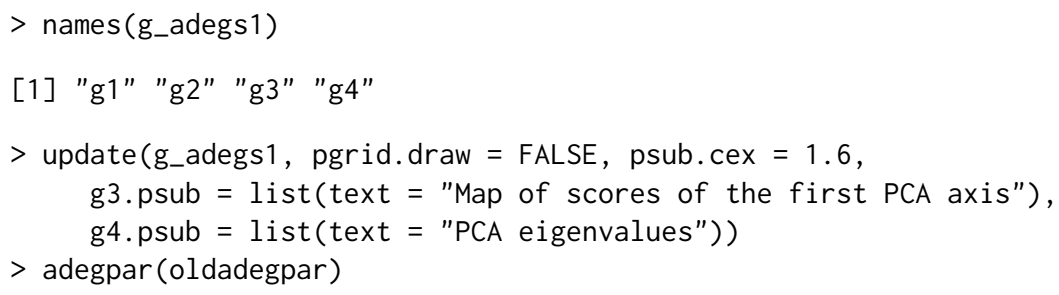




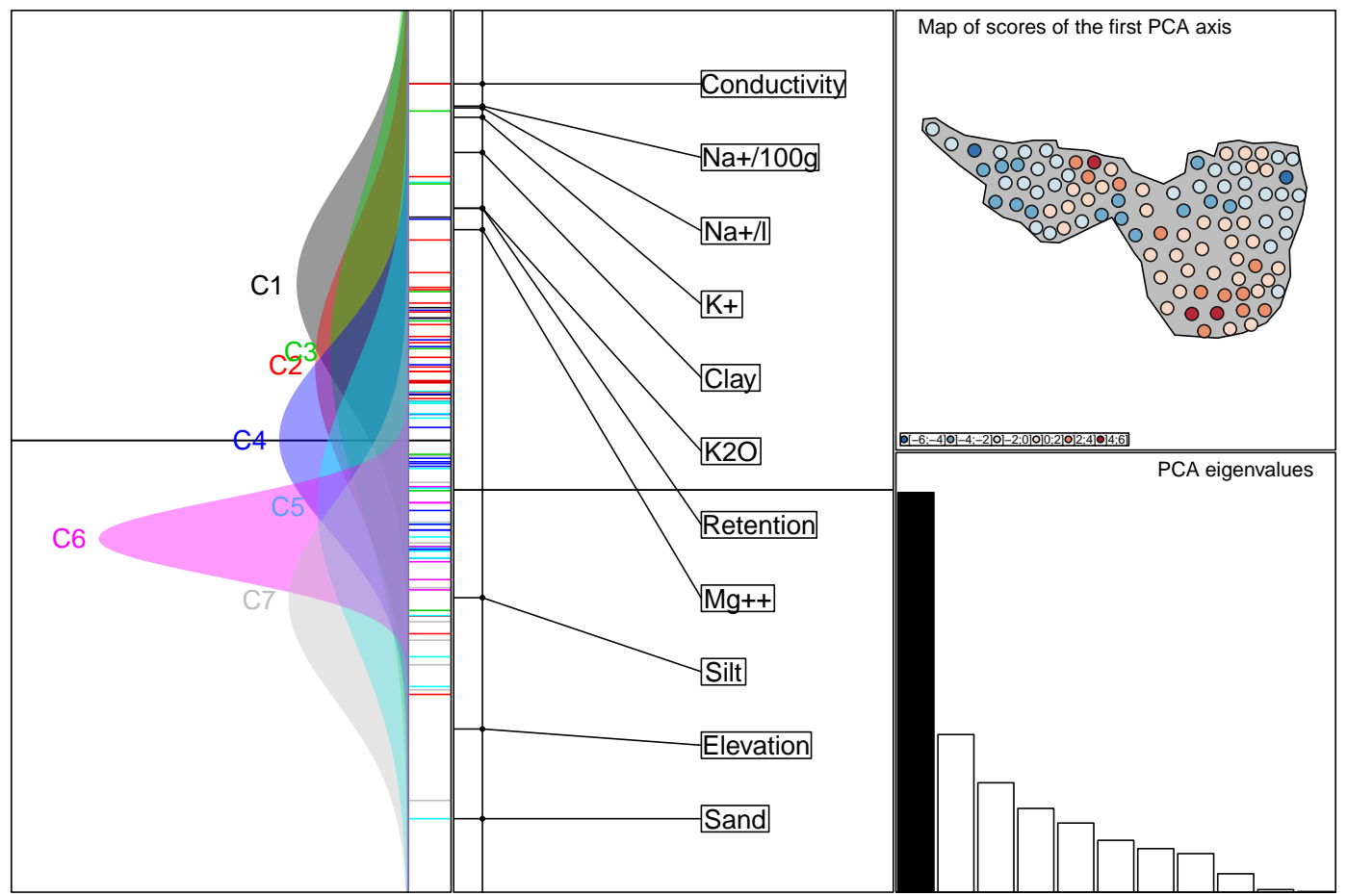

Figure 7: Creation of an "ADEgS" by the manipulation of four "ADEg" objects. Some graphical parameters are globally set before creating the graphs, others are defined when each subgraph is created, and others are updated after the final creation. The left part of the figure is the one-dimensional biplot of the PCA first axis. Gauss curves on the left show the distribution of sampling site PCA scores for the seven ecoregions. The labels on the right show the physico-chemical characteristics of each ecoregion, as evidenced by the PCA. For example, the sampling sites of ecoregion $\mathrm{C} 1$ are characterized by a high conductivity and high levels of salinity, while sites of ecoregions C5, C6 and C7 are higher and more sandy. The top right map recalls that this first axis has a strong spatial structure (see legend to Figure 1), and the bottom right barplot shows that this corresponds to a large part ( $41 \%$ ) of the total inertia.

\section{Multivariate analysis outputs}

All functions formerly available in ade4 to display the outputs of multivariate analyses have been reimplemented in adegraphics. Most of them return an "ADEgS" object.

In our example, the PCA on environmental variables is now applied with the CA weights (coa1\$1w) and stored in pca2. Then, a coinertia analysis is built to identify relationships between environmental variables (pca2) and species distributions (coa1). The results are stored in a coi1 object and are graphically summarized by a call to the plot method.

$>$ pca2 <- dudi.pca(mafragh\$env, row.w = coa1\$lw, scannf = FALSE, $\mathrm{nf}=2$ )

$>$ coi1 <- coinertia(coa1, pca2, scannf = FALSE, $\mathrm{nf}=3$ )

$>$ g_coi $1<-\operatorname{plot}(\operatorname{coi} 1)$

The resulting graphical object (g_coi 1, Figure 8) is an "ADEgS" containing 6 subgraphs displaying the main outputs required to interpret the results.

$>$ length(g_coi1)

[1] 6

In ade4, the graphical outputs provided by the plot function were initially designed to obtain a quick view on the main outputs. However, the number of graphs and their relatively small size did not allow a deep interpretation of the results. Hence, it was often required to write some new $\mathrm{R}$ code to redraw only the useful information in a publication-ready format. In adegraphics, the process is simplified because results are produced as "ADEgS" objects that can be manipulated as standard lists. Each graph is an element of this list and can be easily extracted using the names of elements (for the \$ operator) or their index in the list (for [[]]). The way a graph is created with adegraphics is reachable with the Call attribute of this object. 


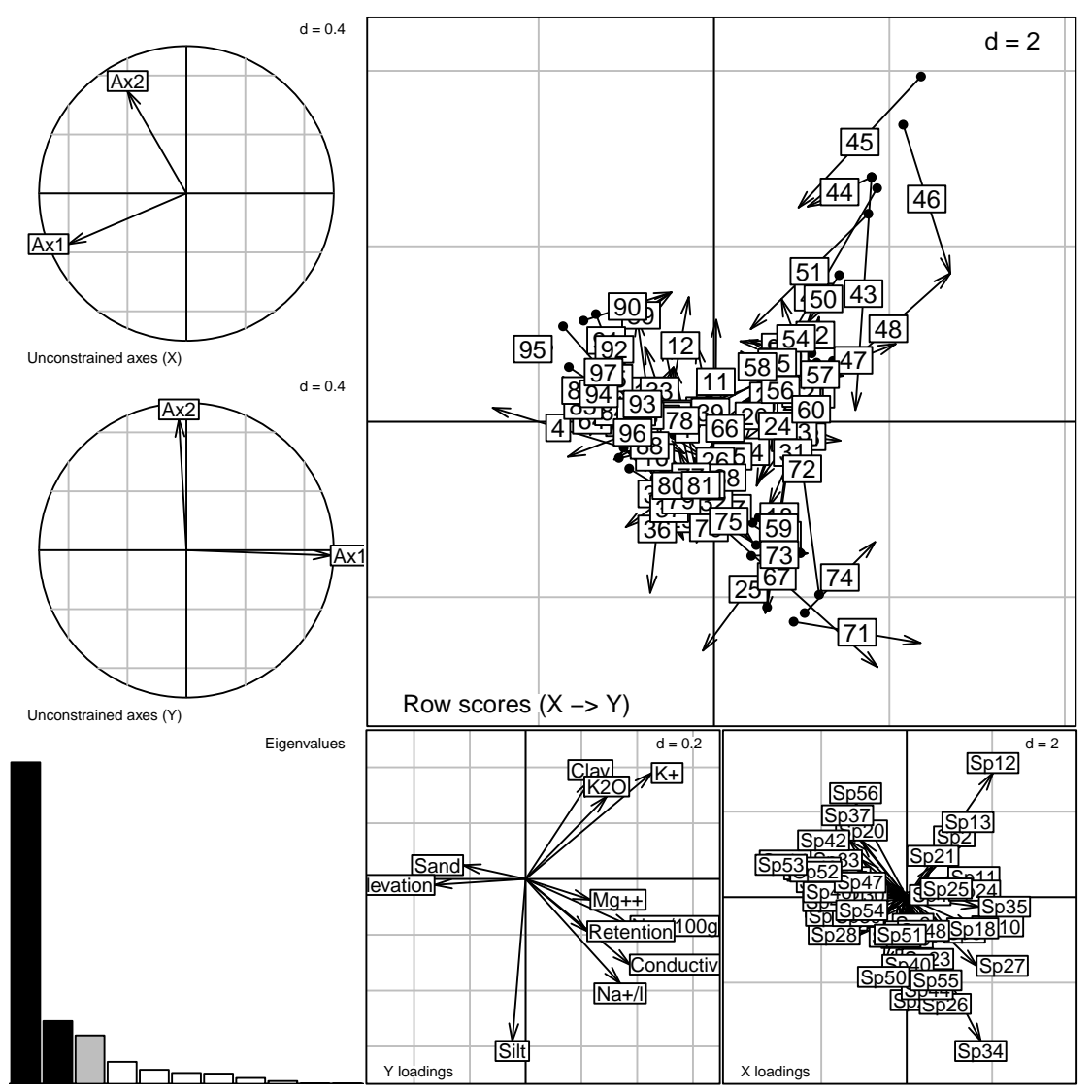

Figure 8: Default representation of a coinertia analysis. The g_coi 1 object is a collection of 6 graphs produced by the plot. coinertia method implemented in adegraphics.

$>$ names(g_coi1)

[1] "Xax" "Yax" "eig" "XYmatch" "Yloadings" "Xloadings"

$>$ g_coi1\$XYmatch \# the same as g_coi1[[4]]

$>$ g_coi1@call

plot. $\operatorname{coinertia}(x=\operatorname{coi} 1)$

$>$ g_coi1\$XYmatch@Call

s. $\operatorname{match}(d f x y 1=\operatorname{coi} 1 \$ m X, d f x y 2=\operatorname{coi} 1 \$ m Y, x a x=1$, yax $=2$, plot $=$ FALSE, storeData $=$ TRUE, pos $=-3$, psub $=\operatorname{list}($ text $=$ "Row scores $(X->Y) ")$, labels $=$ row. names $($ as. data. $f$ rame $(\operatorname{coi} 1 \$ \mathrm{mX}))$, arrows $=$ TRUE, facets $=$ NULL, add $=$ FALSE)

In some subgraphs of Figure 8, information is not easy to read and not necessarily relevant. Thanks to the graphical facilities developped in adegraphics, a new multiple graph can be created to better explore coinertia results.

First, to improve the top-right subgraph (g_coi1\$XYmatch) which is unreadable, a scatter plot is created (g_scl) to represent the scores of sites on the first factorial map of the coinertia, not for each sites (as in g_coi $1 \$ X Y m a t c h$ ) but grouped and colored by ecoregion. To build the new graph, it is usefull to know how g_coi 1 XYmatch was created thanks to its Call attribute (see above). Then the eigenvalues barplot of the coi 1 coinertia (the subgraph called g_coi1\$eig, located at the bottom left of g_coi 1, in Figure 8) is inserted at the topleft of g_scl.

\footnotetext{
$>$ g_scl1 <- s.class $(\operatorname{coi} 1 \$ \mathrm{mX}$, fac $=$ mafragh\$partition, ellipseSize $=0$, chullsize $=0$, starSize $=0.5$, ppoints. cex $=0, \operatorname{col}=c(1: 6,8)$, plot $=$ FALSE $)$

$>$ g_scl2 <- s.class (coi1 $\$ \mathrm{mY}$, fac $=$ mafragh\$partition, ellipseSize $=0$, chullsize $=0$, starSize $=0.5$, ppoints. $\operatorname{cex}=0, \operatorname{col}=c(1: 6,8)$, plot $=$ FALSE $)$
} 
$>$ g_scs1 <- superpose (g_scl1, g_scl2)

$>$ g_sm1 <- s.match(g_scl1@stats\$means, g_scl2@stats\$means, plabels.cex $=0$, plines.lwd $=2$, plot $=$ FALSE)

$>$ g_scl <- superpose (g_scs1, g_sm1)

$>$ g_scl <- insert(g_coi1\$eig, g_scl, posi $=$ "topleft", ratio $=0.3$, plot $=$ FALSE)

Next, the g_coi $1 \$$ Yloadings subgraph is stored in a new g_yload object and then updated: boxes around labels and grid are removed; the subtitle is enlarged.

$>$ g_yload <- g_coi1\$Yloadings

$>$ update(g_yload, plabels.box.draw $=$ FALSE, pgrid.draw $=$ FALSE, psub.cex $=1.2$, plot $=$ FALSE)

Then a g_sl3 object is inspired by the bottom-right subgraph (g_coi1\$Xloadings) of g_coi1: a s. label function is used instead of the s. arrow one, labels are extracted from the mafragh $\$$ spenames $\$$ code vector, labels positions are optimized, points and grid are removed.

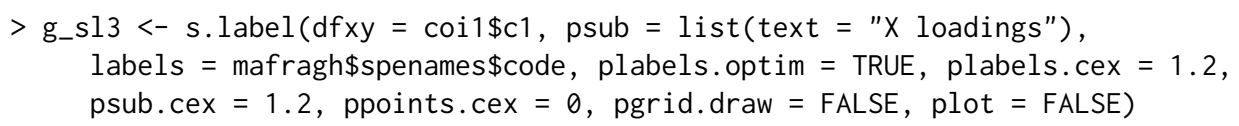

The g_adegs 2 object is at last created with the ADEgS function.

$>$ g_adegs 2 <- ADEgS(list(g_scl, g_yload, g_sl3),

layout $=\operatorname{list}($ mat $=\operatorname{matrix}(c(1,1,2,1,1,3)$, nrow $=2$, byrow $=$ TRUE $)))$

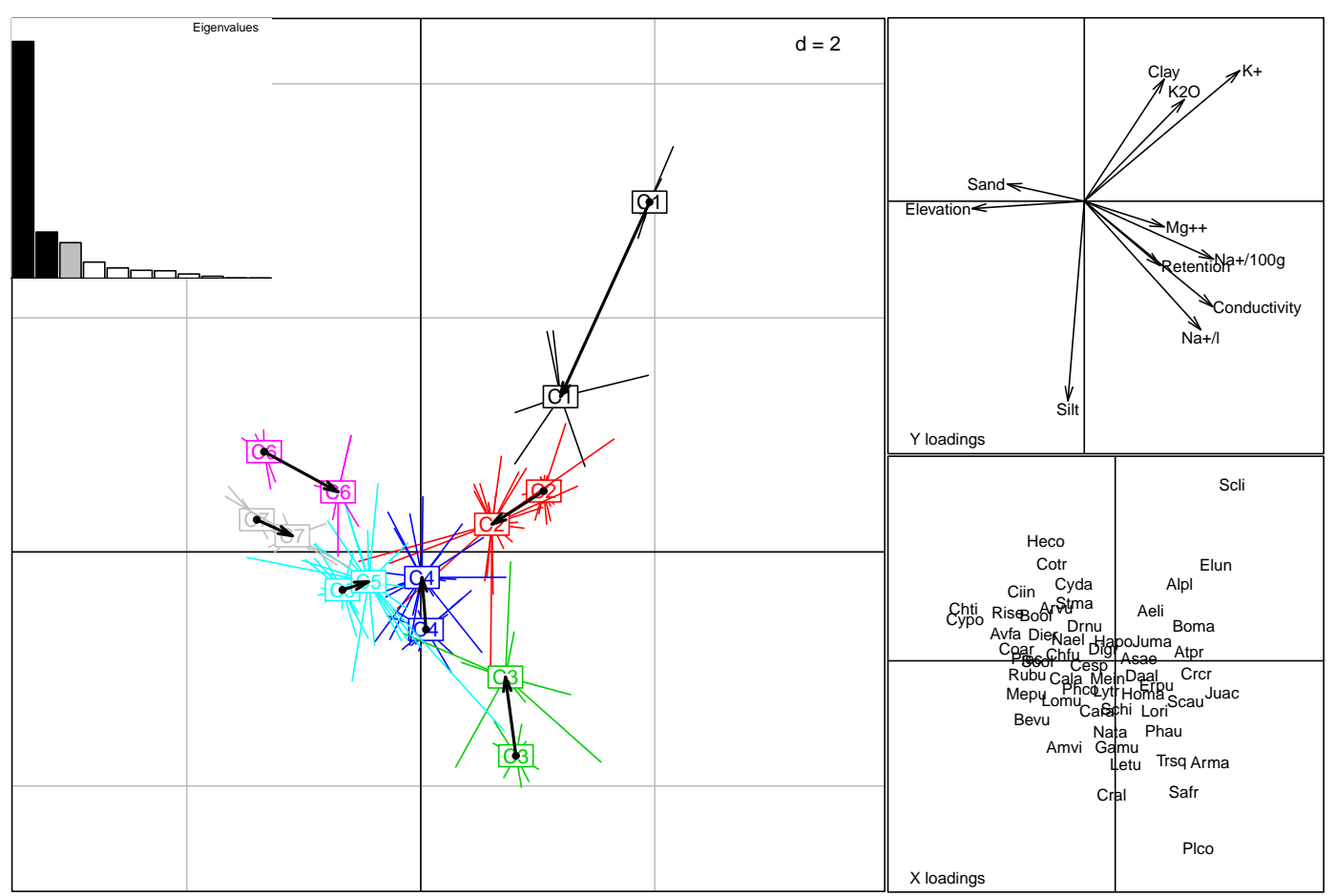

Figure 9: A customized coinertia analysis. The g_adegs2 object is an improvement to the default g_coi1 one. Each graphical parameter can be customized before, during and/or after the object creation and the "ADEgS" building becomes easy. This figure helps interpret the relationships between plant species and environmental parameters. For example, Schoenoplectus Litoralis (Scli) is found preferentially in sites with high clay and potassium rates. On the opposite, Plantago coronopus (Plco) is found in sites with high levels of sodium ions and high conductivity. Cynosurus polybracteatus (Cypo) and Chrozophora tinctoria (Chti) are found in sandy sites with high elevation above sea level. The discrepancy between plant and environmental parameters in the 7 ecoregions is shown in the left graph. For each ecoregion, the coordinates of sites are linked to their gravity center, forming an irregular star. The gravity centers of site coordinates from the plants table and from the environmental parameters table are linked by an arrow. The length of this arrow is therefore representative of the discrepancy between plants and environmental parameters. Regions $\mathrm{C} 1$ and $\mathrm{C} 6$ have to the longest arrows, which means that the discrepancy is higher in these three ecoregions. 


\section{Conclusions}

The adegraphics package is a complete reimplementation of ade4 graphical functionalities with large improvements. The structure of this new package is quite rigorous (formalism provided by S4 classes) but very flexible and easily extendable to support new features. The graphical engine of the lattice package allows to produce high quality graphs for data exploration and publication. This lattice-based implementation provides an efficient and highly-customizable object-based environment to build graphs and it also facilitates the connections between packages. The "ADEgS" class allows to integrate simple "trellis" objects from the lattice package and thus offers the possibility to integrate, in the same graphical object, various graphs created by different packages.

This code is executed with R 3.4.2, ade4 1.7-8 and adegraphics 1.0-8. Other examples are available in the vignette and in the help pages of the package.

\section{Bibliography}

R. S. Bivand and G. Piras. Comparing Implementations of Estimation Methods for Spatial Econometrics. Journal of Statistical Software, 63(18):1-36, 2015. URL https://doi.org/10.18637/jss.v063.i18. [p202]

R. S. Bivand, J. Hauke, and T. Kossowski. Computing the Jacobian in Gaussian Spatial Autoregressive Models: An Illustrated Comparison of Available Methods. Geographical Analysis, 45(2):150-179, 2013a. URL https://doi.org/10.1111/gean.12008. [p202]

R. S. Bivand, E. J. Pebesma, and V. Gomez-Rubio. Applied Spatial Data Analysis with R, Second edition. Springer, NY, 2013b. URL https: //doi .org/10.1007/978-1-4614-7618-4. [p202]

G. de Bélair and M. Bencheikh-Lehocine. Composition et déterminisme de la végétation d'une plaine côtière marécageuse : La Mafragh (Annaba, Algérie). Bulletin d'écologie, 18(4):393-407, 1987. [p199, 204]

S. Dray and A.-B. Dufour. The ade4 Package: Implementing the Duality Diagram for Ecologists. Journal of Statistical Software, 22(4):1-20, 2007. URL https://doi .org/10.18637/jss. v022.i04. [p198]

S. Lê, J. Josse, and F. Husson. FactoMineR: An R Package for Multivariate Analysis. Journal of Statistical Software, 25(1):1-18, 2008. URL https://doi .org/10.18637/jss. v025.i01. [p198]

P. Murrell. R Graphics. Chapman \& Hall / CRC Press, 2005. URL http://www.e-reading.club/ bookreader.php/137370/C486x_APPb.pdf. [p198]

E. Neuwirth. RColorBrewer: ColorBrewer Palettes, 2014. URL https://CRAN.R-project.org/package= RColorBrewer. R package version 1.1-2. [p202]

J. Oksanen, F. G. Blanchet, M. Friendly, R. Kindt, P. Legendre, D. McGlinn, P. R. Minchin, R. B. O'Hara, G. L. Simpson, P. Solymos, M. H. H. Stevens, E. Szoecs, and H. Wagner. vegan: Community Ecology Package, 2017. URL https://CRAN. R-project. org/package=vegan. R package version 2.4-5. [p198]

S. Pavoine, E. Vela, S. Gachet, G. de Bélair, and M. B. Bonsall. Linking patterns in phylogeny, traits, abiotic variables and space: a novel approach to linking environmental filtering and plant community assembly. Journal of Ecology, 99(1):165-175, 2011. URL https://doi org/10.1111/j. $1365-2745.2010 .01743 . x$. [p199]

E. J. Pebesma and R. S. Bivand. Classes and methods for spatial data in R. R News, 5(2):9-13, 2005. URL https://www.r-project.org/doc/Rnews/Rnews_2005-2.pdf. [p202]

D. Sarkar. Lattice: Multivariate Data Visualization with R. Springer, New York, 2008. URL https: //doi.org/10.1007/978-0-387-75969-2. [p198]

W. N. Venables and B. D. Ripley. Modern Applied Statistics with S. Springer, New York, fourth edition, 2002. URL https://doi.org/10.1007/978-0-387-21706-2. [p198]

H. Wickham. ggplot2: Elegant Graphics for Data Analysis. Springer, New York, second edition, 2016. URL https://doi .org/10.1007/978-3-319-24277-4. [p203]

Aurélie Siberchicot

Univ Lyon, Université Claude Bernard Lyon 1, CNRS,

Laboratoire de Biométrie et Biologie Evolutive, 
F-69100, Villeurbanne, France.

ORCiD: 0000-0002-7638-8318

aurelie.siberchicot@univ-1yon1.fr

Alice Julien-Laferrière

Univ Lyon, Université Claude Bernard Lyon 1, CNRS,

Laboratoire de Biométrie et Biologie Evolutive,

F-69100, Villeurbanne, France.

alice.julien. laferriere@gmail.com

Anne-Béatrice Dufour

Univ Lyon, Université Claude Bernard Lyon 1, CNRS,

Laboratoire de Biométrie et Biologie Evolutive,

F-69100, Villeurbanne, France.

ORCiD: 0000-0002-9339-4293

anne-beatrice. dufour@univ-lyon 1.fr

Jean Thioulouse

Univ Lyon, Université Claude Bernard Lyon 1, CNRS,

Laboratoire de Biométrie et Biologie Evolutive,

F-69100, Villeurbanne, France.

ORCiD: 0000-0001-7664-0598

jean. thioulouse@univ-lyon 1.fr

Stéphane Dray

Univ Lyon, Université Claude Bernard Lyon 1, CNRS

Laboratoire de Biométrie et Biologie Evolutive,

F-69100, Villeurbanne, France.

ORCiD: 0000-0003-0153-1105

stephane.dray@univ-1yon 1.fr 